\title{
Efficient Random Process Generation for Reliable Simulation of Complex Systems
}

\author{
Alexey S. Rodionov ${ }^{1}$, Hyunseung Choo ${ }^{2}$, Hee Yong Youn ${ }^{2}$, Tai M. Chung ${ }^{2}$, and \\ Kiheon Park ${ }^{2}$ \\ ${ }^{1}$ Novosibirsk Institute of Computational Mathematics and Mathematical Geophysics \\ Siberian Branch of the Russian Academy of Sceiences, Novosibirsk, Russia \\ alrod@rav.sscc.ru \\ ${ }^{2}$ School of Electrical and Computer Engineering \\ Sungkyunkwan University, Suwon, Korea \\ $\{$ choo, youn,tmchung,khpark\}@ece.skku.ac.kr
}

\begin{abstract}
Generating pseudo random object is one of the key issues in computer simulation of complex systems. Most earlier systems employ independent and identically distributed random variables, while those of real processes often show nontrivial autocorrelation. In this paper a new approach is presented that generates random process for given marginal distribution and autocorrelation function. The proposed approach achieves significant speed-up and accuracy by using truncated distribution instead of order statistics. An experiment displays more than ten times speed-up for reasonable size system. Moreover, the proposed generator is simple and requires less memory.
\end{abstract}

\section{Introduction}

In developing a stochastic model to describe a real system, a model realistically replicating the actual operation should be chosen. Even though mathematical analysis is a useful tool for predicting the performance of a system wherever it is tractable, computer simulation is gaining more attention due to the relatively recent advent of fast and inexpensive computational power. It allows faithful reproduction of the behavior of real processes and comprehensive analysis based on the data collected.

For computer simulation of complex systems pseudo random objects of various nature need to be generated [12. In most general purpose discrete simulation independent and identically distributed random variables are usually used. However, the random numbers of real processes often show nontrivial autocorrelation. For example, observe that the two processes of a same exponential marginal distribution $(\lambda=1)$ in Figure 1 look quite different due to different autocorrelation functions. For an $\mathrm{M} / \mathrm{M} / 1$ queue with the service rate of 1.5 and inter-arrival rate of 1 , the average waiting time is 7.94. For the inter-arrival distribution of Figure $1(\mathrm{a})$ and (b), it is 12.14 and 7.62, respectively. The difference is significant based on the $t$-criteria with a significance level of $95 \%$. This exemplifies the importance of simulation with dependent random numbers.

V.N. Alexandrov et al. (Eds.): ICCS 2001, LNCS 2074, pp. 912-921 2001.

(C) Springer-Verlag Berlin Heidelberg 2001 
A number of researches related to this problem have been done, while they are for generating mostly correlated vectors and fields [3-6]. The method commonly employed for generating dependent random vectors [37], however, is not suitable for long one-dimensional sequence due to the requirement on large size memory. As a result, other methods were developed to generate stable one-dimensional processes with some given properties and arbitrary length [8-10]. Based on the theoretical foundation on generating random processes with required properties [10 12], we propose a new method for generating pseudo random processes with a given marginal distribution and autocorrelation function (ACF). Such processes often allow us to faithfully replicate the actual operation of the simulated system. For a 16-state randomized Markov chain about 14 times acceleration is achieved in comparison with the previous algorithm [12, while the storage requirement is much smaller. Moreover, the proposed generator is simple.

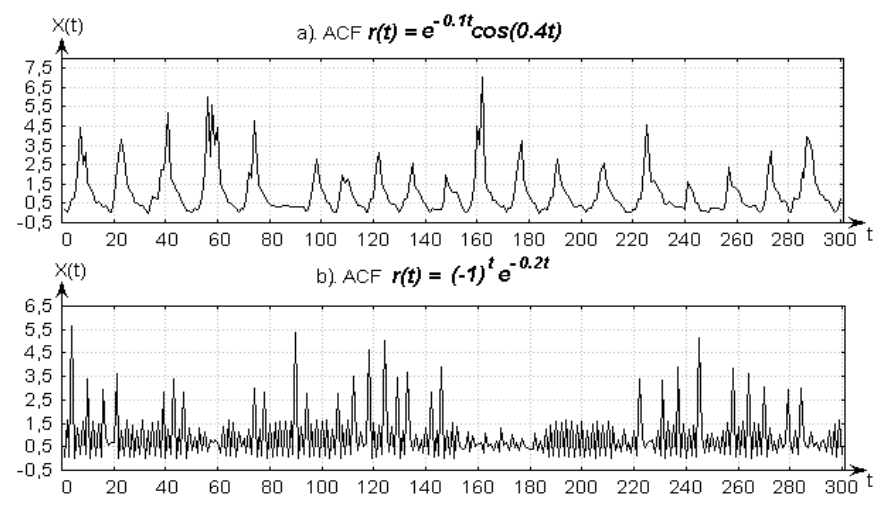

Fig. 1. Two different processes with the same marginal distribution.

\section{Basic Concepts and Notation}

Let $\xi_{1}, \xi_{2}, \ldots, \xi_{N}$ be a set of independent random variables with identical continuous distribution $F(x)$, and let $\xi_{(1)}, \xi_{(2)}, \ldots, \xi_{(N)}$ be the same set of random variables ranked in non-decreasing order. It is assumed that the ordering rule does not change by time as

$$
P\left(\xi_{(i)}(t)<x\right)=P\left(\xi_{(i)}<x\right)=F_{i}(x) .
$$

We obviously have

$$
\sum_{i=1}^{N} F_{i}(x)=N \times F(x) .
$$


Let $\Psi$ be a homogeneous Markov chain independent of $\xi_{i}(t)$ with a set of states $i=1,2, \ldots, n$, a vector of initial probabilities, $\pi_{0}$, and two-stochastic matrix of transition probabilities:

$$
\begin{aligned}
& \left(P\left(\Psi_{t+1}=j \mid \Psi_{t}=i\right)\right)=\left(p_{i j}\right)=P \\
& \sum_{i=1}^{N} p_{i j}=1 ; \quad \sum_{j=1}^{N} p_{i j}=1 ; \quad i, j=1,2, \ldots, N .
\end{aligned}
$$

Let the process

$$
\xi_{t}=\xi_{\Psi_{t}}(t), t=0,1,2, \ldots
$$

be a randomized Markov chain (RMC).

If the initial probabilities of Markov chain are $\pi_{0}=(1 / N, \ldots, 1 / N)$, then the finite-dimensional distribution of the process is:

$$
\begin{aligned}
P\left(\xi_{t_{1}}<x_{1}, \ldots, \xi_{t_{m}}<x_{m}\right)= & 1 / N \times \sum_{\substack{\left.j_{1}, \ldots, j_{m}=1 \\
j_{j_{1}}\left(t_{2}-t_{1}\right) \times \ldots \times p_{j_{m-1} j_{m}}\left(t_{m}-t_{m-1}\right)\right] .}}^{N}\left[F_{j_{1}}\left(x_{1}\right) \times \ldots \times F_{j_{m}}\left(x_{m}\right) \times\right.
\end{aligned}
$$

The marginal distribution and ACF of randomized Markov chain above are:

$$
\begin{aligned}
& F_{\xi}(x)=1 / N \times \sum_{i=1}^{N} F_{i}(x)=F(x), \\
& r^{\prime}(\tau)=\frac{1}{N D_{\xi}} \sum_{i, j=1}^{N} M_{i} M_{j}\left(p_{i j}^{\prime}(\tau)-\frac{1}{N}\right), \quad \tau=1,2, \ldots
\end{aligned}
$$

where $D_{\xi}$ is variance of the marginal distribution and $M_{i}$ is the expectation of order statistics $\xi_{(i)}$ as:

$$
M_{i}=\int_{-\infty}^{\infty} x d F_{i}(x) .
$$

To obtain $d F_{i}(x)$ of the expression above we use the well-known expression for probability elements of order statistics [11]:

$$
\begin{aligned}
d F_{i}(x) & =\frac{\Gamma(n+1)}{\Gamma(i) \Gamma(N-i+1)} F^{i-1}(x)[1-F(x)]^{N-i} f(x) d x \\
& =\frac{N !}{(i-1) !(N-i) !} F^{i-1}(x)[1-F(x)]^{N-i} f(x) d x \\
& =(N-i+1)\left(\begin{array}{c}
N \\
i-1
\end{array}\right) F^{i-1}(x)[1-F(x)]^{N-i} f(x) d x
\end{aligned}
$$

Based on this we obtain an algorithm generating pseudo random process of a given distribution. It consists of two parts: obtaining a new state of the Markov chain and generating a random number according to the corresponding distribution. 
The algorithm for simulating a Markov chain with a given matrix of transition probabilities is well-known. The following is the algorithm proposed for generating a Markov chain [9].

\section{Algorithm G1.}

Input: $N$ is the number of states of the Markov chain; $n$ is the required length of the process realized.

Output: an array $X(1 \times n)$ of a process realization. The algorithm consists of the following steps:

Step 1 (Preliminary). Put a sample of a non-correlated sequence with the given marginal distribution $F(x)$ into an urn with reset. The counter $k$ is set to one. The initial state of Markov chain is gained uniformly from $1,2, \ldots, N$.

Step 2. From an urn, $\xi_{i}(i=1, \ldots, N)$ are extracted by a random fashion and then ranked in non-decreasing order. Let denote resulting variables as $\xi_{(i)}(i=1, \ldots, N)$.

Step 3. By the given two-stochastic matrix of transition probabilities the next state (say $j$ ) of the Markov chain is generated.

Step 4. $\xi_{j}$ is copied to output as $X_{k}$, and $k$ is increased by one.

Step 5. If $k>n$, the generation comes to an end. Otherwise, go to Step 2.

Since Algorithm G1 has several shortcomings [13, we propose an improved algorithm.

\section{Algorithm G2.}

Input and output are same as for Algorithm G1.

Step 1 (Preliminary). The initial state of Markov chain is gained uniformly from $1,2, \ldots, N$. The counter $k$ is set to one.

Step 2. By the given two-stochastic matrix of transition probabilities the next state (say $j$ ) of the Markov chain is generated.

Step 3. Generation of pseudo random number, $\xi$, by a truncated distribution $F_{j}(x)$ on $j$-th inter-fractile interval. $\xi$ is copied to output as $X_{k}$, and $k$ is increased by one.

Step 4. If $k>n$, the generation comes to an end. Otherwise, go to Step 2.

The cumulative distribution function $F_{i}(x)$ whose distribution is truncated on interval $\left(x_{i-1}, x_{i}\right)$ is

$$
F_{i}(x)= \begin{cases}0, & \text { for } x<x_{i-1} \\ \frac{F(x)-F\left(x_{i-1}\right)}{F\left(x_{i}\right)-F\left(x_{i-1}\right)}, & \text { for } x_{i-1} \leq x<x_{i} . \\ 1, & \text { for } x \geq x_{i}\end{cases}
$$

If $x_{i}$ is $i$-th fractile of the marginal distribution, we obtain a simpler expression

$$
F_{i}(x)= \begin{cases}0, & \text { for } x<x_{i-1} \\ N\left(F(x)-\frac{i-1}{N}\right), & \text { for } x_{i-1} \leq x<x_{i} . \\ 1, & \text { for } x \geq x_{i}\end{cases}
$$


The Expressions (2) and (3) are still true, but now $M_{i}$ ought to be obtained using the distribution (5), so:

$$
M_{i}=\int_{x_{i-1}}^{x_{i}} x d F_{i}(x)=\left.x F_{i}(x)\right|_{x_{i-1}} ^{x_{i}}-\int_{x_{i-1}}^{x_{i}} F_{i}(x) d x=x_{i}-\int_{x_{i-1}}^{x_{i}} F_{i}(x) d x
$$

For obtaining the transition probability matrix we need to solve the system (3) of nonlinear equations of degree $\tau$. The solutions were found only for some special kinds of two-stochastic matrix 10, whereas it is necessary to have a general solution for real problems that could be done only numerically. We next present how to determine transition probability matrix.

\section{Determination of Transition Probability Matrix}

In determining transition probability matrix, we need to minimize the difference between real ACF and ACF of simulated process by the elements of the two stochastic matrices. If we use the sum of squares of the differences as a distance, then we have to solve the following optimization task:

$$
\begin{gathered}
\Phi(\tau)=\sum_{\tau}\left(r(\tau)-r^{\prime}(\tau)\right)^{2} \rightarrow \min \\
r^{\prime}(\tau)=\frac{1}{N D_{\xi}} \sum_{i, j=1}^{N} M_{i} M_{j}\left(P_{i j}^{\prime}(\tau)-\frac{1}{N}\right) \\
\sum_{i=1}^{N} P_{i j}^{\prime}=1 ; \quad \sum_{j=1}^{N} P_{i j}^{\prime}=1 ; \quad i, j=1,2, \ldots, N \\
\forall i, j \quad 0 \leq P_{i j}^{\prime} \leq 1,
\end{gathered}
$$

where $P_{i j}^{\prime}(\tau)$ is the element of the $i$-th row and $j$-th column of the derivable matrix in power $\tau \cdot r^{\prime}(\tau)$ is the $\mathrm{ACF}$ of a randomized Markov chain corresponding to the matrix and given marginal distribution.

Molchan had stated [9] that the gradient optimization method is not suitable for solving the optimization problem of Expressions (7) and (8). Instead, he offered a heuristic method of stochastic optimization and a modification of it. Our experiments with various optimization methods revealed that the simple gradient method of "Quickest descent" with the use of penalty functions is the best, and thus it is adopted here.

First of all, let us free the task from the restrictions of two-stochasticity, Expression (8), by using the following substitutions:

$$
\begin{aligned}
P_{i N}^{\prime} & =1-\sum_{j=1}^{N-1} P_{i j}^{\prime}, \quad P_{N i}^{\prime}=1-\sum_{j=1}^{N-1} P_{j i}^{\prime}, \quad i=1, \ldots, N-1, \\
P_{N N}^{\prime} & =\sum_{i, j=1}^{N-1} P_{i j}^{\prime}-N+2 .
\end{aligned}
$$


Then we can formally transform our optimization problem to a problem of unconstrained optimization by adding a penalty function:

$$
\begin{gathered}
Q\left(P_{i j}^{\prime(k)}, \rho_{i j}^{(l)}, \delta_{i j}^{(m)}\right)=\Phi\left(P_{i j}^{\prime(k)}\right)+\sum_{i, j=1}^{N-1}\left[\rho_{i j}^{(l)} U_{i j}^{(k)}\left(P_{i j}^{\prime(k)}\right)^{2}+\delta_{i j}^{(l)} V_{i j}^{(k)}\left(P_{i j}^{\prime(k)}-1\right)^{2}\right] \\
i, j=1, \ldots, N-1
\end{gathered}
$$

where

$U_{i j}^{(k)}=\left\{\begin{array}{ll}1 & \text { if } P^{\prime}{ }_{i j}<0, \\ 0 & \text { otherwise }\end{array}\right.$ (index operator)

$V_{i j}^{(k)}=\left\{\begin{array}{ll}1 & \text { if } P^{\prime}{ }_{i j}>1, \\ 0 & \text { otherwise }\end{array}\right.$ (index operator),

$\rho_{i j}^{(l)}=10^{l}$ (weight coefficients), where $l$ is the amount of iterations from the moment of $P_{i j}^{\prime(k)}<0, l \leq 100 ;$

$\delta_{i j}^{(m)}=10^{l}$ (weight coefficients), where $m$ is the amount of iterations from the moment of $P_{i j}^{\prime(k)}>1, l \leq 100$.

Derivation $d Q / d p_{k l}(1 \leq k, l \leq N)$ is defined by the following expressions (hereafter we will omit $(k)$ and apostrophe at $P$ for better readability of expressions):

$$
\begin{aligned}
\frac{d Q}{d p_{k l}} & =\frac{d \Phi}{d p_{k l}}+2 \sum_{i, j=1}^{N-1}\left[\rho_{i j}^{(l)} U_{i j} P_{i j}-\delta_{i j}^{(l)} V_{i j}\left(P_{i j}-1\right)\right] \\
\frac{d \Phi}{d p_{k l}} & =\frac{1}{N D_{\xi}} \sum_{\tau}\left[\frac{1}{N D_{\xi}} \sum_{i, j=1}^{N} M_{i} M_{j}\left(P_{i j}(\tau)-\frac{1}{N}\right)-r(\tau)\right] \sum_{i, j=1}^{N} M_{i} M_{j} \frac{d P_{i j}(\tau)}{d p_{k l}}
\end{aligned}
$$

In [13], we show how the computation steps can be minimized. Experiments display very fast access to the minimum area in about 3 to 5 steps and abrupt deceleration of convergence. The deceleration rate greatly depends on the length of ACF; it is almost imperceptible for $n<10$, while advancement to optimum slows down very much for $n>30$. This might be due to the cancellations in the calculation of multiple sums and products of the numbers of very small magnitudes. The number of operations grows exponentially with $\tau$. Use of special numerical methods and high-precision calculations could improve the situation. In case when high accuracy is needed, we propose the following optimization scheme.

Step 1. Input the initial two-stochastic matrix, say unitary matrix or matrix with equal elements.

Step 2. Find a matrix for ACF with a length restricted to a small number.

Step 3. Using the result of the previous step, find a matrix for ACF with the needed length.

Step 4. If the accuracy is not sufficient, use random optimization for more precise result. 
For our experiments we choose one of the worst cases in the point of view of the difference from the examples given in [10]. Here the marginal distribution is exponential with $\lambda=1, N=10$, and ACF $r(\tau)=e^{-0.1 \tau} \cos (0.4 \tau)$,

The difference displayed by the algorithm above with 2500 random steps was about $10 \%$. Higher accuracy can be achieved using the proposed optimization scheme. First, by sequential execution of the gradient optimization for an ACF length from 5 to 40 with step 5 , we obtain a difference of $9.6 \%$ in not more than 10 steps for each length. Consequent execution of 500 steps of random search with one-dimensional optimization gives us the difference of $6.1 \%$. The computation time was also significantly reduced as about 3 minutes on the $600 \mathrm{MHz}$ Pentium III PC. If the number of states is increased to 16 , then a difference $2.4 \%$ is archived for the ACF, but the computation takes more than 20 minutes. The difference with gradient minimization is $14.6 \%$. We next compare Algorithm G1 and G2 for generating random processes with exponential marginal distribution.

\section{Case Studies}

Algorithm G1 According to (4) the distribution density of $\xi_{i}$ in our case is

$$
f_{i}(x)=\lambda(N-i+1)\left(\begin{array}{c}
N \\
i-1
\end{array}\right)\left(1-e^{-\lambda x}\right)^{i-1} e^{-\lambda(N-i+1) x},
$$

and, consequently, the expectation for $\xi_{i}$ is

$$
\begin{aligned}
M_{i}= & \int_{0}^{\infty} x f_{i}(x) d x=\int_{0}^{\infty} x \lambda(N-i+1)\left(\begin{array}{c}
N \\
i-1
\end{array}\right)\left(1-e^{-\lambda x}\right)^{i-1} e^{-\lambda(N-i+1) x} d x= \\
& \frac{1}{\lambda}(N-i+1)\left(\begin{array}{c}
N \\
i-1
\end{array}\right) \int_{0}^{\infty} t\left(1-e^{-t}\right)^{i-1} e^{-(N-i+1) t} d t= \\
& \frac{1}{\lambda}(N-i+1)\left(\begin{array}{c}
N \\
i-1
\end{array}\right) \sum_{j=0}^{i-1} \frac{(-1)^{i-j-1}}{N-j}\left(\begin{array}{c}
i-1 \\
j
\end{array}\right) \int_{0}^{\infty}(N-j) t e^{-(N-j) t} d t .
\end{aligned}
$$

The last integral is expectation of the exponential distribution with parameter $N-j$. Hence the final result for $M_{i}$ is:

$$
M_{i}=\frac{N-i+1}{\lambda}\left(\begin{array}{c}
N \\
i-1
\end{array}\right) \sum_{j=0}^{i-1} \frac{(-1)^{i-j-1}}{(N-j)^{2}}\left(\begin{array}{c}
i-1 \\
j
\end{array}\right)=\frac{K_{i}}{\lambda},
$$

where $K_{i}$ is a coefficient that depends only on the values of $i$ and $N$. N is decided as some not very large constant independent on the distribution parameter $\lambda$. When $N$ is fixed, all $K_{i}$ 's can be easily calculated.

Algorithm G2 The cumulative distribution function $F_{i}(x)$ is determined by Expression (5) and expectations $M_{i}$ by Expression (6). For the exponential marginal distribution considered, the $i$-th fractile of exponential distribution is equal to

$$
x_{i}=-\frac{1}{\lambda} \ln \left(\frac{N-i}{N}\right), 1 \leq i \leq N-1,
$$


where $N$ is the number of intervals (for the uniformity of expressions, we also add the leftmost and rightmost values: $x_{0}=0$ and $x_{N}=\infty$ ). We obtain an expression for the cumulative distribution function of truncated exponential distribution on the interval between the fractiles from Expressions (5) and (11).

$$
F_{i}(x)=N\left(1-e^{-\lambda x}\right)-i+1 .
$$

The corresponding expectations are

$$
\begin{aligned}
M_{i}= & \frac{1}{\lambda}\left(1+\ln \frac{N(N-i)^{N-i}}{(N-i+1)^{N-i+1}}\right)= \\
& \frac{1}{\lambda}(1+\ln N+(N-i) \ln (N-i)-(N-i+1) \ln (N-i+1))=\frac{K_{i}^{*}}{\lambda}, \\
& i=1, \ldots, N-1 \\
M_{N}= & \frac{1}{\lambda}(1+\ln N)=\frac{K_{N}^{*}}{\lambda}
\end{aligned}
$$

As for Algorithm G1, the expectation $M_{i}$ has inverse negative relationship with the distribution parameter $\lambda$. Note that calculating $K_{i}^{*}$ 's in Algorithm G2 is much simpler than calculating $K_{i}$ 's in Algorithm G1, i.e., it does not require to calculate the sums and binomial coefficients.

For the generation process we need to produce the samples from the total population with exponential distribution for Algorithm G1, while from the truncated interval $\left[x_{i}-1, x_{i}\right)$ for Algorithm G2. For detail, refer to [13]. As the Algorithm G2 does not need the sorting step, it is much faster than G1. For generating 100,000 numbers for a 16-state randomized Markov chain, $1.86 \mathrm{sec}$ was taken with Algorithm G2. It was $25.04 \mathrm{sec}$ with Algorithm G1, which is more than 13 times slower than the proposed approach. The accuracy of the proposed ACF approximation scheme is illustrated in Figure 2. Observe that the difference with the proposed algorithm is smaller than that of Algorithm G1. In Figure 3, we illustrate an example of ACF

$$
r(\tau)= \begin{cases}1-0.05 \tau & \text { if } 0<\tau \leq 20 \\ 0 & \text { otherwise }\end{cases}
$$

which shows the significance of proper choice of maximal ACF length, $n$. If we have $n=20$, the ACF is well approximated up to $\tau=20$ but the difference becomes large beyond that point. For $n=25$, we achieve better result, and $n=30$ allows a really good approximation. Yet on the interval $[1, \ldots, 20]$, all approximations are almost equally good. In this example, the number of states, $N$, is 16 .

\section{Conclusion}

In this paper we have proposed an algorithm for generating randomized Markov chains with given marginal distribution and ACF. The proposed approach achieves significant speed-up and accuracy by using truncated distribution instead of order statistics. Randomized Markov chains can approximate different 

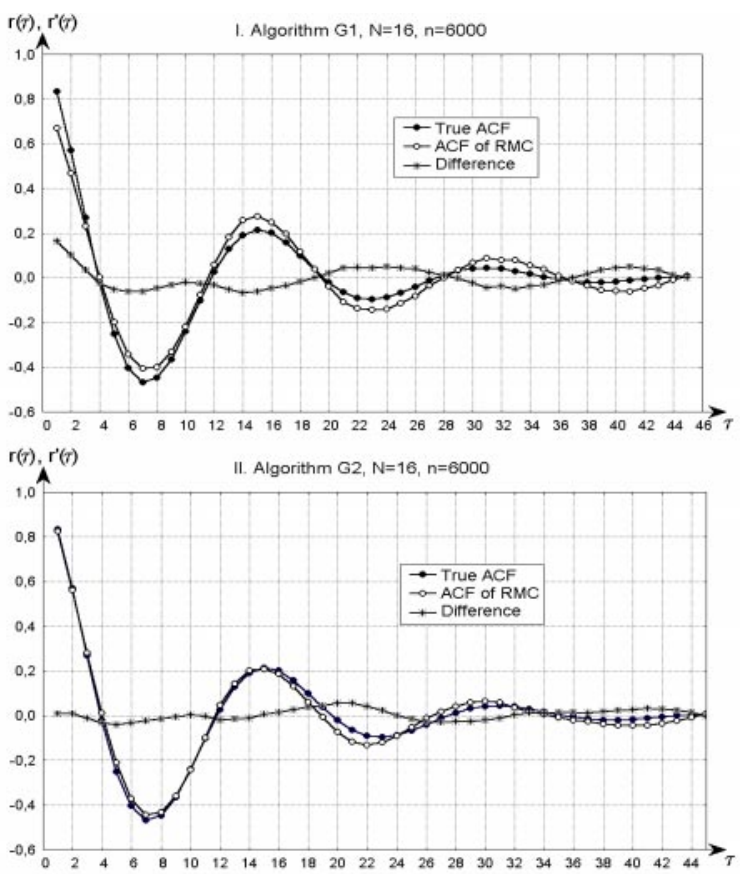

Fig. 2. Comparison of given and obtained ACFs.

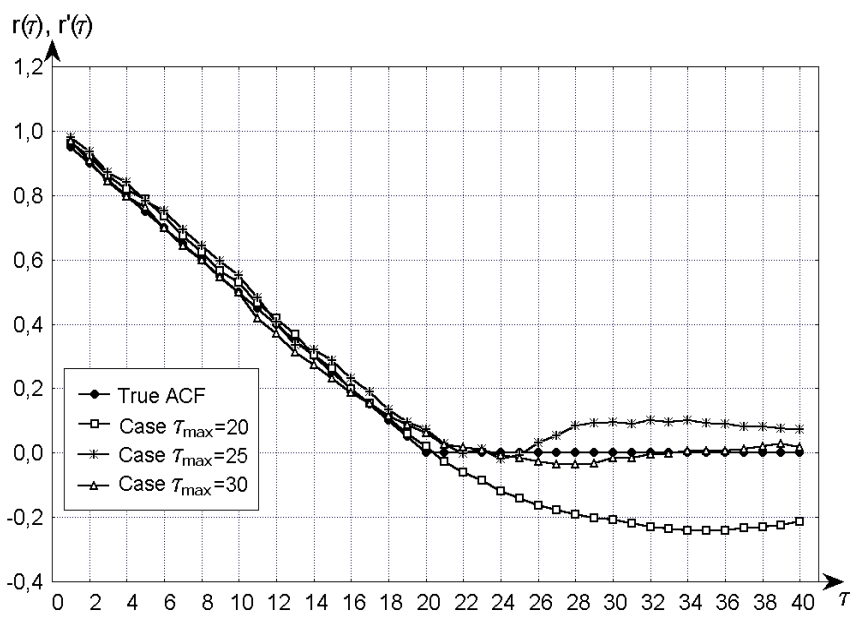

Fig. 3. Influence of an $\mathrm{ACF}$ length truncation on the approximation accuracy (based on algorithm G2). 
stationary random processes when only the marginal distribution and ACF are taken into account. The accuracy of an ACF approximation is good enough if the numbers of the states of the Markov chain is not large. Usually, not more than 20 states provide a sufficiently small difference between the approximated ACF and that of the randomized Markov chain. On the other hand, with the number of states more than 20, it is almost impossible to complete the optimization task for the transition probability matrix on modern PC in reasonable time. We have also presented an optimization approach using penalty functions. In the future researches we will try to improve the quality of optimization. A significant improvement will also be achieved by using parallel algorithms for matrix computation.

Acknowledgement. This work was supported in part by Brain Korea 21 project and grant No. 2000-2-30300-004-3 from the Basic Research Program of Korea Science and Engineering Foundation.

\section{References}

1. Al-Shaer, E., Abdel-Wahab, H., Maly, K.: HiFi: A New Monitoring Architecture for Distributed Systems Management. The 19th IEEE Int'l Conf. on Distributed Computing Systems (1999) 171-178

2. Gribaudo, M., Sereno, M.: Simulation of Fluid Stochastic Petri Nets. Proceeding of the 8th MASCOTS (2000) 231-239

3. Dagpunar, J.: Principle of Random Variate Generation. Clarendon Press, Oxford (1988)

4. Blacknell, D.: New Method for the Simulation of Correlated $\mathcal{K}$-distributed Clutter. IEE Proc. Rad., Son. Nav., Vol. 141 (1994) 53-58

5. Ronning, G.: A Simple Scheme for Generating Multivariate Gamma Distributions with Non Negetive Covariance Matrix. Technometrics, Vol. 19 (1977) 179-183

6. Bustos, O.H., Flesia, A.G., Frery, A.C.: Simulation of Correlated Intensity SAR Images. Proc. XII Brazilian Symp. on Comp. Graph. and Image Proc. (1998) 10

7. Hammersley, J., Handscomb, D.: Monte-Carlo Methods. Methuen\&Co, (1964)

8. Letch, K., Matzner, R.: On the Constraction of a Random Process with Given Power Spectrum and Probability Density function. Proceedings of the 1998 Midwest Symposium on Systems and Circuits, Vol. 41 (1999) 14-17

9. Molchan, S.I., Prelovskaja, A.A., Rodionov, A.S.: Program Complex for Generation of Random Processes with Given Properties. System modeling, Trans. of Computing Center of Siberian Branch of Academy of Sciences of USSR, Novosibirsk, ISSN 0134-630X, No. 13 (1998) 70-81

10. Molchan, S.I.: About One Approach to Generation of Random Processes with Given Properties. Simulation of Computing Systems and Processes, Perm's State Univercity, USSR (1986) 59-72

11. Wilks, S.: A Mathematical Statistics. Second edition, John Wiley\&Sons (1962)

12. Feller, W.: An Introduction to Probability Theory and its Applications. John Wiley\&Sons (1971)

13. Rodionov, A.S., Choo, H., Youn, H.Y., Chung, T.M., Park, K.: On Generating Random Variates for Given Autocorrelation Function. TR-2001-1, School of ECE, Sungkyunkwan University (2001) 